\title{
THE GROWTH OF HERPETOLOGY IN THE PUERTO RICO AND VIRGIN ISLAND AREA
}

\author{
Chapman Grant, Major, U. S. Army.
}

The earliest writers, Navarette, Oviedo, Casas and Herrera, writing in the latter part of the 15th century make no mention of our reptiles, according to de Armas in his "La Zoologica de Colon y de los Primeros Exploradores de America". They mention reptiles in Santo Domingo and on the Costa Firme only.

1654. Dutertre treats of the reptiles and amphibians of the Lesser Antilles and the Virgin Islands as a whole. He was probably plagiarized by de Rochefort in 1658 and later. Their description of the iguana; its habits and its use by the indians is graphic and probably authentic.

1788. Fra Inigo Abbad y Lasierra speaks of three species as occurring in Puerto Rico. This is the first mention of Puerto Rican reptiles.

1793. H. West wrote on the reptiles of St. Croix and St. Thomas, listing 7 species.

1796-98. Mr. Andre-Pierre Le Dru, a botanist, recorded 12 species from Puerto Rico, but no scientific cognizance is given to his herpetological work.

1863. J. Reinhardt and C. F. Leuthen published the first list of any scientific value, recognizing 10 species of reptiles and amphibians.

1868. E. D. Cope listed 15 species from Puerto Rico.

1876. W. Peters recognized 21 species.

1882. Agostin Stahl published his Catalogue. At this time 23 species were known.

1904. Dr. Leonard Stejneger recorded 35 species in a most scholarly work.

1928. K. P. Schmidt and in 1930 Thomas Barbour recognized 43 species from the Puerto Rico Area and an additional 10 from the Virgin Islands.

1932. The present list comprises 62 species from the Puerto Rico Area and 28 from the Virgin Islands of which 13 species occur on parts of both groups; a total of 77 species exclusive of marine turtles, but inclusive of extinct and introduced forms. The writer has described 11 new species in this Journal, one in Copeia and several remain to be described in my collection of 7,200 specimens. 
402 THE JOURNAL OF THE DEPARTMENT OF AGRICULTURE OF P. R.

KEY TO THE FOLLOWING LISTS:

$\mathrm{n}$ - New species described by the writer.

$f$ - First local record of a known species.

$\mathrm{r}$ - Reestablished as a valid species.

z - Extinct locally or wholly.

? - Local occurrence questioned. If in first column the validity of species is questioned.

i - Introduced.

Some of the data in the following lists was kindly furnished by Miss Doris Cochran. 
Herpetological list of species from the Puerto Rico Area

Bufo lemur.

B. turpis.

B. marimus i

Leptodactylus albilabris

L. Fallax i

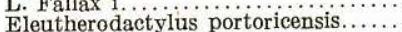

$\mathrm{E}$. gryllus.

E. locustus. .

E. cramptoni .

E. antillensis.

E. brittoni.

E. wightmani.

E. richmondi...

E. monensis.

E. unicolor

E. karlschmidti .

E. cochranae.

E. cooki.

E. lentus

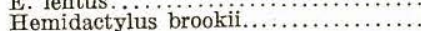

H. mabouia.

Thecadactylus rapicaudus

Phyllodactylus pulcher.

Sphaerodactylus grandisquamis

S. macrolepis.

S. klauberi....

S. roosevelti.

S. nicholsi.

S. townsendi

S. danforthi

$\mathrm{S}$. monensis.

S. gaigeae.

Iguana iguana

Anolis cuvieri.

A. roosevelti...

A. cristatellus.

A. acutus.

A. gundlachi

A. evermanni.

A. pulchellus

A. krugi.

A. poncensis.

A. monensis.

A. newtonii ?...

Cyclura stejnegeri.

C. mattea $Z$.

C. portoricensis $\mathrm{Z}$

C. pinguis

Celestus pleii...........

Ameiva wetm

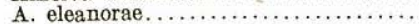

A. polops.

A. exsul.

A. alboguttata

A. birdorum

Amphisbaena caeca.

A. fenestrata.

Mabuya sloanii.

M. semitaeniatus.

Typhlops sp. 8.

T. richardii.

T. monensis ?

T. rostellatus.

Epicrates inornatus.

$$
\text { E. sp. } 9 \text {. }
$$

E. monensis.

Dromicus stahli

D. exiguus.

Alsophis antillensis

A. portoricensis.

A. variegatus ?

A. sancti-crucis...

Pseudemys rugosa

P. descussata.

Testudo tabulata $\mathrm{i}$.

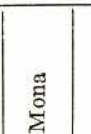

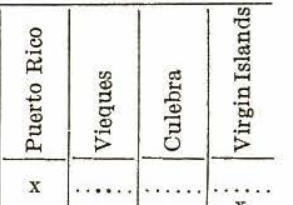

Cope................ 1868 .

Barbour.................... 1917

Linnaeus.............1758

Gunther.................

Müller ............... 1923

Schmidt. . . . . . . . . 1927

Schmidt. . . . . . . . . 1920.

Schmidt. . . . . . . . 1920.

Schmidt. . . . . . . . . . 1920

R. \& L............. 1863

Schmidt. . . . . . . . . 1920

Schmidt............. 1920.

Stejneger...............1904

Meerwarth............1901.

Stejneger..............1904. .

Grant............... 1931

Grant................ 1932

Grant.................1932

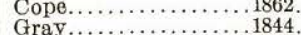

Jonnes................1868

(Houthuyn) .........1782.

Gray.................. 1830 .

Stejneger................. 1904.

Gunther..............1859

Grant................1931.

Grant.................

Grant.................. 1931

Grant................1931.

Grant.................1931.

Meerwarth...............

Linnaeus..............1758.

Meerem..............1820.

Grant.................

Hallowell . ............1856

Peters .................1871

Cope...................1861

Stejneger............. 1904

D. \& B.............. 1837

Peters............... 1871

Stejneger...............1904

Stejneger.............. 1904

Gunther..............1859.

Barbour \& N..........1916.

Miller...................

Barbour................... 1919.

Barbour.................1916.

D \& B . . . . . . . . . . 1839

Stejneger.................

Grant \& Rooseveit...1931.

Cope \& R............ 1862

Cope...............1862.

Boulenger.................

Grant................. 1932 .

Cuvier . ...............1829.

Cope................1861

Stejneger..................

Daudin................1803.

Wiegmann ..........

Shaw $\ldots \ldots \ldots \ldots \ldots \ldots \ldots \ldots 1844$.

Schmidt................

Stejneger...............1904.

Reinhardt............1843

Grant....................

Zenneck................ 1898 .

Stejneger...............1904.

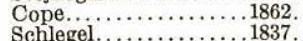

R. \& L................

Sehmidt .................. 1926

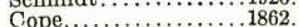

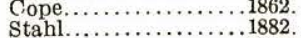

Peters.

Walbaum
1876.

1782 . 


\section{Notes :}

1. The Puerto Rico form seems to be distinct from that of the Virgin Islands.

2. Introduced by the Experiment Station of Mayagüez in 1929 again in 1932 from Dominica without evidence of establishment as yet.

3. I believe this name includes two species.

4. Misidentified by earlier writers as being $H$. mabouia.

5. This species has recently been confused with $S$. grandisquamis and $S$. monensis.

6. Known only from Caja de Muertos island.

7. Known only from Diablo Key off Fajardo.

8. This group of the genus is in need of revision. I hope to report on my series of over 100 from 7 islands.

9. To be described.

In the Virgin Island List:

St. Croix includes Buck Island.

St. Thomas includes; Water Island, Hassell Island, Buck Island Saba Island.

St. John includes; Congo and Lovango Keys.

St. James includes; Little St. James, Dog Island.

Tortola includes; Guana, Buck, Salt and Peter Islands.

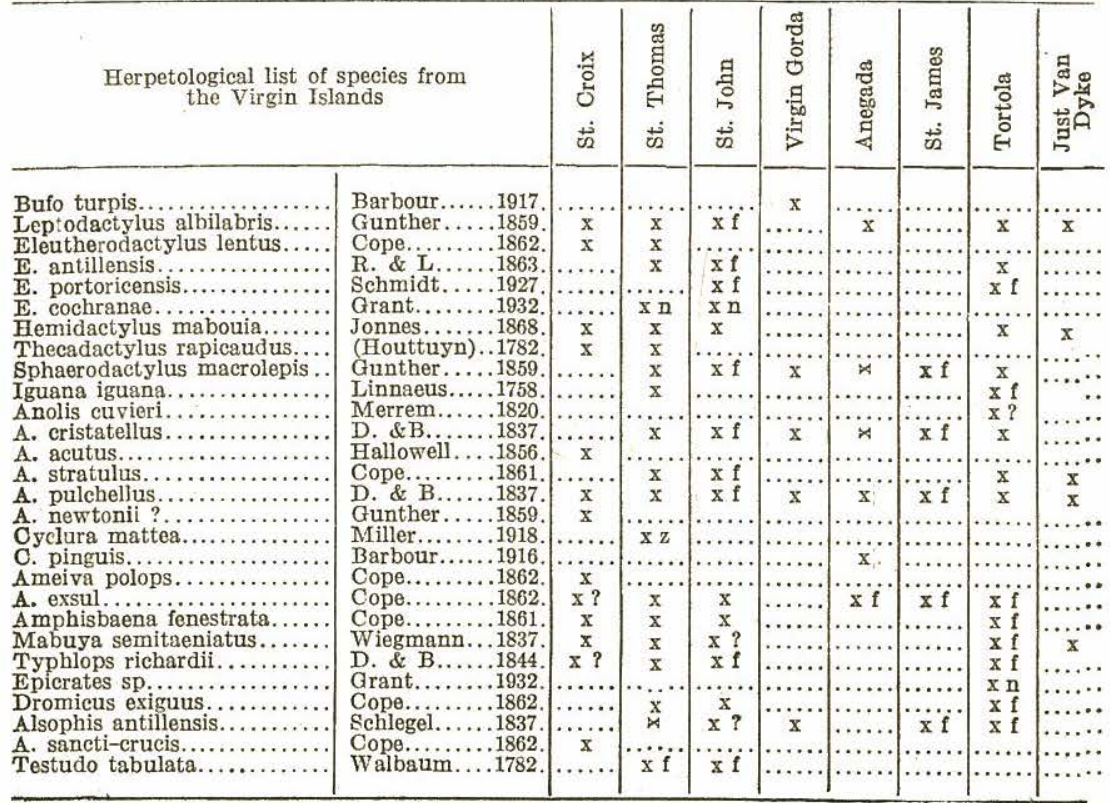

